\title{
Analysis of expressed sequence tags from Trypanosoma cruzi amastigotes
}

\author{
Gustavo C Cerqueira ${ }^{++}$, Wanderson D DaRocha ${ }^{++}$, Priscila C Campos, \\ Cláudia S Zouain, Santuza MR Teixeira+
}

\author{
Departamento de Bioquímica e Imunologia, ICB, Universidade Federal de Minas Gerais, Av. Antônio Carlos 6627, 31270-010 \\ Belo Horizonte, MG, Brasil
}

A total of 880 expressed sequence tags (EST) originated from clones randomly selected from a Trypanosoma cruzi amastigote cDNA library have been analyzed. Of these, 40\% (355 ESTs) have been identified by similarity to sequences in public databases and classified according to functional categorization of their putative products. About $11 \%$ of the mRNAs expressed in amastigotes are related to the translational machinery, and a large number of them (9\% of the total number of clones in the library) encode ribosomal proteins. A comparative analysis with a previous study, where clones from the same library were selected using sera from patients with Chagas disease, revealed that ribosomal proteins also represent the largest class of antigen coding genes expressed in amastigotes (54\% of all immunoselected clones). However, although more than thirty classes of ribosomal proteins were identified by EST analysis, the results of the immunoscreening indicated that only a particular subset of them contains major antigenic determinants recognized by antibodies from Chagas disease patients.

Key words: Trypanosoma cruzi - gene expression - amastigotes - antigens

Chagas disease, caused by the protozoan Trypanosoma cruzi, is a parasitic disease, which affects about 16 million people in Latin America. Although vectorial and transfusional transmission in most countries is now under control, chronic $T$. cruzi infections still result in serious morbidity and mortality, mainly due to heart tissue damage observed in a significant percentage of chronically infected individuals (WHO, www.who.ch).

T. cruzi has three main developmental stages. Epimastigotes multiply extracellularly in the midgut of the reduviid bug vector and migrate to the hindgut, where they differentiate into non-dividing metacyclic trypomastigotes, that are excreted with the insect feces. Infective trypomastigotes, inoculated into the mammalian host through mucous membranes and skin cuts, invade a number of cell types and differentiate into amastigotes, which multiply intracellularly in the cytoplasm of the parasitized cell. After a certain number of cell divisions, amastigotes transform into trypomastigotes that enter the circulatory system when the host cell lyses. Bloodstream trypomastigotes invade new host cells and can be transmitted to a reduviid bug during a blood meal where they differentiate again into epimastigotes and complete the life cycle (Brener 1973).

Financial support: $\mathrm{CNPq}$

${ }^{+}$Corresponding author and fellowship from Capes. E-mail: santuzat@mono.icb.ufmg.br

${ }^{++}$Both author have contributed equally to the work.

The sequence data reported herein has been deposited in GenBank under accession numbers listed at http:// www.icb.ufmg.br/ nage/aest/supplemental_table.html.

Received 10 February 2005

Accepted 19 May 2005
Knowledge of the gene expression pattern of each developmental stage is a valuable contribution to the study of the parasite biology and the pathogenesis of Chagas disease. Expressed sequence tags (EST) analysis is a powerful approach that rapidly expands the knowledge not only towards gene discovery but also regarding stage specific gene expression profiles. EST data has also been useful in the final steps of genome annotation and gene prediction of whole genome sequencing projects. Beginning in 1994, the T. cruzi EST sequencing project has generated more than 10,000 ESTs, mainly derived from a normalized cDNA library constructed with RNA from epimastigote cultures (Brandão et al. 1997, Verdun et al. 1998, Porcel et al. 2000) and, more recently, from trypomastigotes and amastigotes of the CL Brener strain (Agüero et al. 2004) (http://cruzi.cgb.ki.se/ TcruziEST/index.html). After an intense effort initiated by an International Consortium of three sequencing centers (The Institute for Genomic Research, TIGR, Seattle Biomedical Research Institute, and Karolinska Institute), the complete genome sequence of the $60 \mathrm{Mb}$ haploid genome of the CL Brener clone is expected to be published shortly.

We have been interested in studying genes expressed in amastigotes, particularly the ones encoding amastigote antigens, which may be more relevant to the chronic chagasic pathology and are likely to be more useful as a source for antigenic targets for serodiagnosis and vaccine development (Matsumoto et al. 1993, Low et al. 1998, Boscardin et al. 2003). To generate more information regarding the amastigote gene expression profile and to identify new candidate targets for vaccine studies, we isolated cDNA clones derived from an amastigote library immunoselected with sera from patients with Chagas disease (DaRocha et al. 2002). Here we compared the results obtained in this previous study with the analysis of 880 EST originated from randomly chosen clones from the same cDNA library as well as with other T. cruzi EST 
datasets. Based on sequence comparisons, we report the putative functions of several novel T. cruzi genes expressed in amastigotes. The EST analyses also showed that clones encoding ribosomal proteins, which have been previously identified as one of the main targets for the humoral immune response of patients with Chagas disease (DaRocha et al. 2002), also constitute the largest class of cDNAs identified in the amastigote library.

\section{MATERIALS AND METHODS}

cDNA library - The unidirectional amastigote cDNA library was constructed in the bacteriophage $\lambda$-ZAPII according to the procedures indicated in the cDNA synthesis and in vitro phage packaging kit (Invitrogen), as described by Teixeira et al. (1994). Amastigote cells of the Tulahuén strain were obtained from cultures of RA786 cell line culture two weeks after infection and purified by centrifugation in metrizamide gradient also described by Teixeira et al. (1994). Based on rRNA and microsatellite typing, the Tulahuén strain belongs to the same T. cruzi II lineage of the clone CL Brener that was chosen for the genome project (SMR Teixeira, unpublished). In vivo excision protocol using the Escherichia coli strain XL-1 Blue was conducted with an aliquot of phage supernatant according to the cDNA library manual (Invitrogen). Transformed bacteria were plated in medium containing $\mathrm{X}$-gal and about 1200 individual colonies, identified as $\beta$ galactosidase negative, were randomly picked, inoculated into 96 deep well microtiter plates and submitted to doublestrand plasmid DNA preparation according to previously published protocols (Vasconcelos et al. 2003).

Nucleotide sequencing - Plasmid DNA was sequenced in 96-well microplates with "DYEnamic ${ }^{\mathrm{TM}} \mathrm{ET}$ dye terminator cycle sequencing (MegaBACE ${ }^{\mathrm{TM}}$ )" and the MegaBACE 1000 capillary sequencer (Amersham Pharmacia Biotech). Single-pass sequencing reactions were performed on each template with M13 Forward and Reverse primers, and sequences longer than 100 bases were further analyzed.

Sequence analyses - The chromatogram produced for each clone was analyzed by a software developed by us, named AEST (stands for Análise de ESTs de Tripanosomatídeos, in portuguese). AEST is a web-based application, which automates the processing of ESTs through the integration of several softwares that are routinely used for DNA sequence analyzes. AEST executes a pipeline consisting of the following softwares: (1) phred, for base-calling processing; (2) cross_match, for screening vector sequences; (3) BLASTCLUST, for clustering sequences; and (4) BLASTX and BLASTN for searching similar sequences in the GenBank database (Altschul et al. 1990, Erwing et al. 1998). In the current EST analysis, phred was executed with trim parameter turned on, trim value 0.18 ; cross_match penalty was adjusted to -2 and minscore to 14. BLASTX and BLASTN queries were made against GenBank nonredundant database with the default parameter, except from the low complexity filter, which was turned off. BLASTCLUST executions were performed with the following parameters: -L 0.7 (length coverage), -S 96 (score density), and -W 40 (word size). After automated analysis, the resulting sequences were manually inspected with the following objectives: to assign, if possible, a putative product according to the results of the similarity search (BLASTX and BLASTN) and to remove from further analyses low quality sequences or sequence contaminants, such as ribosomal RNA sequences. Each sequence with a positive identification product was classified according to its probable function in one of the following functional categories: cell cycle, RNA, and DNA synthesis; metabolism; protein destination; translation machinery; structural and cytoskeletal; signal transduction; transporter and cellular trafficking; and those sequences that do not fit in none of the previous categories were grouped in the "others" class. All 880 ESTs were submitted to the dbEST database but can also be accessed through the supplemental table available at http:// www.icb.ufmg.br/ nage/aest/supplemental_table.html.

\section{RESULTS AND DISCUSSION}

Single-pass sequencing was performed from either the 5 ' or 3'-end of 1152 cDNA clones randomly picked from an unidirectional, non-normalized cDNA library constructed with poly-A (+) RNA isolated from $T$. cruzi amastigotes. After removal of low-quality sequences and vector sequences, a total of 880 ESTs were submitted to blast searches against the GenBank nucleotide database and assembled into clusters. The average length of reliable sequence was $510 \mathrm{nt}$. The T. cruzi SL sequence was detected in 19 of the selected clones and a poly-A tail was found in 379 of them. A total of 151 sequences (17\% of the clones analyzed) were assembled into 53 clusters, whereas 729 ESTs represent singletons. The largest cluster contains 18 ESTs, and 12 clusters were found to contain more than 5 ESTs (see below). This clustering analysis indicates that the redundancy of the library estimated from the percentage of clones assembled into clusters is relatively low, considering the levels of redundancy reported by Porcel et al. (2000) (54\%) and Verdun et al. (1998) (28\%) for cDNAs libraries constructed with RNA derived from T. cruzi epimastigotes.

Almost $60 \%$ of the sequences (525 ESTs) did not show any significant similarities to sequences deposited in GenBank. It is thus possible that, even with a small-scale sequencing survey, a significant number of novel T. cruzi genes can be identified. Similarly to other reports of EST analyses $0.4 \%$ of the clones contain ribosomal RNA sequences, but a relatively large proportion (3\%) contains known $T$. cruzi repetitive elements. The repetitive sequences identified were mainly SIRE repetitive elements (Vazquez et al. 1994), which have also been reported in previous analyses of epimastigote libraries. Stable transcripts derived from repeated elements in T. cruzi have been identified by several groups. It has been speculated that they may be involved with reshaping of the genome, or that they may affect the expression of neighboring genes by influencing trans-splicing reactions (Vasquez et al. 1994).

Fig. 1 shows the distribution among eight functional categories of all ESTs to which a putative identity could be assigned using Blast analyses. Similar to the EST distribution obtained in previous EST studies (Verdun et al. 


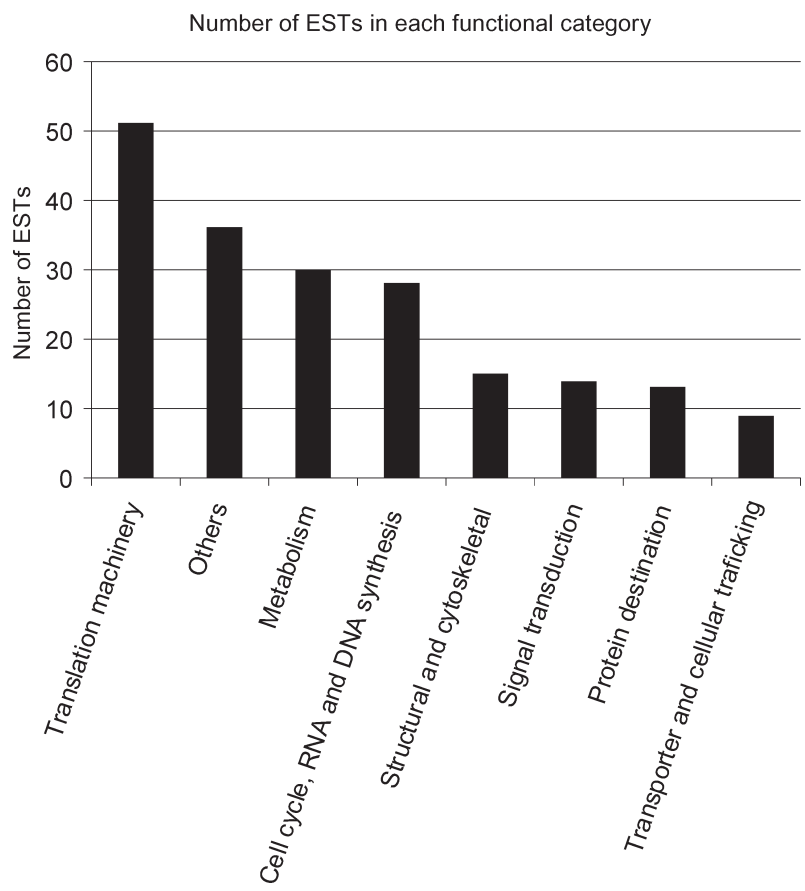

Fig. 1: functional distribution of amastigote expressed sequence tags (ESTs). The graph includes only non-redundant ESTs and shows the number of ESTs classified in each of the eight functional categories, according to the Blast search analysis. The category "others" comprises ESTs whose products could not be classified into any of these categories and includes detoxification enzymes, such as tryparedoxin and membrane and cell surface proteins. In the "protein destination" category we included proteases, proteasomes, chaperonins, and heat shock proteins. The full list of the identified ESTs and a detailed functional classification can be found at http:/ /www.icb.ufmg.br/ nage/aest/supplemental_table.html

1998, Porcel et al. 2000, with epimastigote cDNA libraries and Agüero et al. 2004, with an amastigote cDNA library), a large fraction of mRNAs encoding components of the translation machinery is found in amastigotes. Because the two amastigote libraries were not normalized, we expected a significant number of cDNAs to correspond to abundant transcripts present in this stage of the parasite. In addition to ribosomal proteins, cDNAs encoding histones are among the most abundant sequences in the amastigote libraries. ESTs encoding histone $\mathrm{H} 3$ constitute the largest cluster we identified ( $2 \%$ of the total number of clones, Table) and were also detected as highly abundant cDNAs in the amastigote library described by Aguiero et al. (2004) (corresponding to $1.2 \%$ of the total number of clones). Histone $\mathrm{H} 3$ was identified as the 2 nd and 3rd most frequently identified EST in the two epimastigote cDNA libraries ( 1 and $0.6 \%$, in the libraries described by Porcel and Verdun's group, respectively) as well as an abundant sequence in the trypomastigote cDNA library reported by Agüero et al. (2004). It should be noted that, in contrast to other eukaryotes, histone transcripts from trypanosomatids are poly-adenylated, and thus, will be part of the cDNA population generated from oligo-dT primed reverse transcription reactions. Such high frequency of ESTs encoding histones in the epimastigotes and amastigotes libraries is in accordance with northern blot analyses performed by Recinos et al. (2001) who shows that steady state histone mRNA levels are much higher in dividing epimastigotes and amastigotes, as compared to non-dividing trypomastigotes. It is however surprising that the ESTs derived from the trypomastigote library described by Agüero et al. (2004) also showed a relatively high proportion of histone sequences.

Besides histones and ribosomal proteins, sequences homologous to cytochromes, and heat-shock proteins are among the largest clusters identified in the amastigote EST dataset (Table). We also identified a sizeable number of clones derived from large $T$. cruzi gene families such as mucins and trans-sialidase (GP85), known to be expressed in amastigotes (Acosta-Serrano et al. 2001, Frasch 2001). As expected, we identified the amastigote-specific amastin mRNA as one of the six most abundant cDNA clusters in the amastigote library. This frequency is consistent with northern blot results showing a 50-fold increase of the levels of amastin mRNA expression in amastigotes, as compared to the other two forms of the parasite (Teixeira et al. 1994). A complete list of all ESTs is available at http:/ /www.icb.ufmg.br/ nage/aest/supplemental_table.html.

\section{TABLE}

Most abundant amastigote expressed sequence tags (ESTs)

\begin{tabular}{ll}
\hline Putative product & Number of ESTs
\end{tabular}

Histone H3 18

Ribosomal protein S9 15

Cytochrome-c oxidase chain III 13

Heat shock protein $90 \quad 8$

$\begin{array}{ll}\text { Cytochrome b } & 7\end{array}$

GP85

Histone H2A 6

Amastin 6

Ribosomal protein S12 5

Elongation factor 1-alpha 5

Mucins 5

Casein kinase 1 isoform $2 \quad 5$

The high frequency of ribosomal protein cDNAs $(9 \%$ of the total number of cDNAs) is in agreement with the results of the amastigote EST survey reported by Agüero et al. (2004), who found an even larger percentage (17.4\%) of total ESTs generated from the CL Brener amastigote library encoding ribosomal proteins. This proportion is also similar in the data set from the two epimastigote cDNA libraries (Verdun et al. 1998, Porcel et al. 2000), thus indicating that both forms of $T$. cruzi depend on the maintenance of the protein synthesis apparatus characteristic of rapidly dividing cells. In line with that, microarray studies of gene expression during trypomastigote (a non-dividing stage) to amastigote differentiation also identified several genes encoding ribosomal proteins as a group of amastigote up-regulated genes (Minning et al. 2003). The most abundant mRNAs encoding ribosomal proteins expressed in amastigotes are S9 and S12. A cluster encoding the $T$. cruzi homologue of the $\mathrm{S} 12$ ribosomal protein, 
which may present and additional extra-ribosomal function in the cell (Coetzee et al. 1994), was also identified as one of the most predominant cDNAs of the amastigote library described by Agüero et al. (2004).

Such high prevalence of clones encoding ribosomal proteins was also evident in our previous survey of the same amastigote cDNA library, using an immunoscreening protocol. Ribosomal proteins accounted for the large majority $(54 \%)$ of all clones that react with a pool of sera from Chagas disease patients (DaRocha et al. 2002). Previous literature reports have indicated that ribosomal proteins constitute one of the main targets of the humoral immune response against infections not only by $T$. cruzi but also by other parasites such as Leishmania chagasi and Plasmodium falciparum (Levin et al. 1989, Skeiky et al. 1994, Chatterjee et al. 2000). This is in contrast to histone proteins, which are recognized as a prominent antigen in Leishmania and Plasmodium falciparum infections but not by $T$. cruzi infected individuals (Requena et al. 2000). Interestingly, similar to ribosomal proteins, histones also form nucleoprotein complexes and are also encoded by abundant mRNAs in amastigotes. Ribosomal P proteins are one of the most extensively studied $T$. cruzi antigens because antibodies directed against them, found in sera from patients with chronic Chagas heart disease, cross react with host proteins. Unlike other eukaryotes, T. cruzi possesses four types of $\mathrm{P}$ protein genes TcP0, $\mathrm{TcP} 1, \mathrm{TcP} 2 \alpha$, and TcP2 $\beta$, each of which is present in multiple copies in the genome (Skeiky et al. 1994). The epitopes recognized by antibodies present in sera from Chagas disease patients, located at the C-terminal region of the $T$. cruzi ribosomal P proteins, share sequence homology with human P proteins and $\beta$-adrenergic receptors (Levin et al. 1989, Kaplan et al. 1997). As shown in Fig. 2, in the immunoscreening survey, we found four classes of ribosomal proteins reacting with sera from Chagas disease patients, among them, the previously characterized $T$. cruzi ribosomal $\mathrm{P}$ proteins $\mathrm{TcP} 0$ and $\mathrm{TcP} 2 \beta$. In addition, we iden- tified the orthologues of two eukaryotic ribosomal proteins L19 and L7a, named TcRpL19 and TcRpL7a, as highly immunogenic. Thus, in contrast to the limited number of ribosomal sequences showing antigenic reactions, the EST data indicated that a much larger variety of ribosomal protein mRNAs are highly expressed in amastigotes, since ESTs encoding a total of 34 classes of ribosomal proteins were identified. While TcRpL19 and TcRpL7a are encoded by abundant mRNAs, corresponding, each, to $5 \%$ of the total number of ESTs containing ribosomal protein sequences, cDNAs encoding TcP0 and TcP $2 \beta$ were not detected in our EST survey.

One way to explain why TcRpL7a and TcRpL19 are highly immunogenic is the fact that they contain repeated amino acid motifs. The repetitive Ala, Lys, and Pro-rich motifs are similar, but not identical, and are positioned at the N-terminal (TcRpL7a) or the C-terminal (TcRpL19) of the polypeptide chains (DaRocha et al. 2002). Interestingly, these repetitive sequences are not found in the orthologous genes of any other organism including $T$. brucei and Leishmania. Further studies using recombinant versions of the full-length protein and truncated forms containing only the repetitive and the non-repetitive portions of TcRPL 7a, have demonstrated that the repetitive sequence is responsible for the reactivity of this antigen with sera from Chagas disease patients (Pais et al. 2002). It will be interesting to determine how the presence of this repetitive sequence affects the three-dimensional structure of these proteins as well as the overall structure of the ribosome. A search for repetitive motifs in other $T$. cruzi ribosomal proteins using the available CL Brener genomic database (www.genedb.org) showed that amino acids repeats are also present in ribosomal proteins $\mathrm{S} 2$, S10, and L23a, but only L23a has a repetitive motif similar to TcRpL7a and TcRpL19. Amino acid repeats have been recognized as a recurrent theme since the earlier studies characterizing $T$. cruzi and other parasite antigens, but their role still remains a subject of intense speculation
A

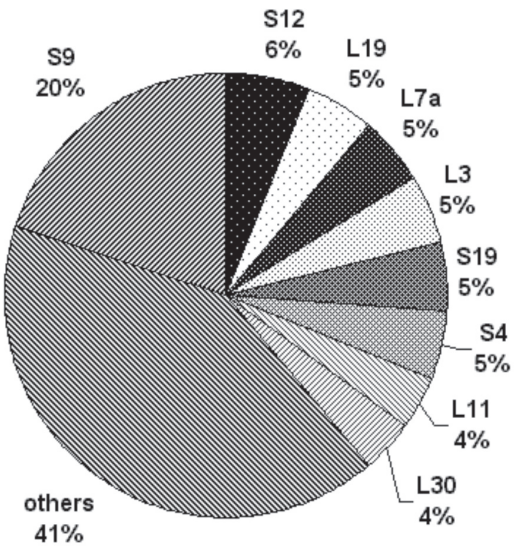

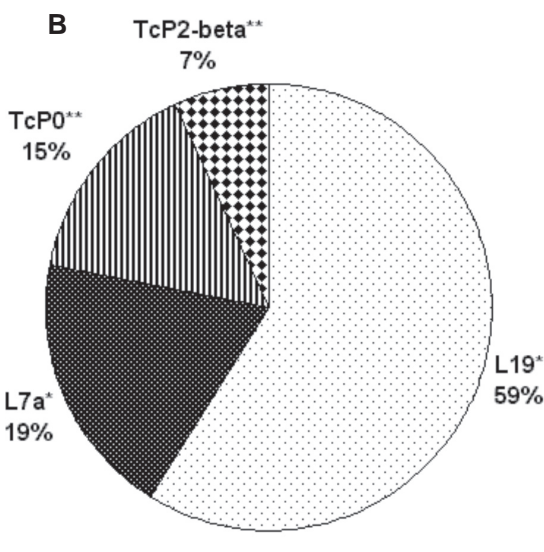

Fig. 2: comparison of expressed sequence tag (ESI) and immunoscreening approaches for the identification of cDNAs encoding ribosomal proteins in the amastigote library. cDNA clones containing sequences homologous to ribosomal proteins that were identified by the EST analysis (A) and immunoscreening the amastigote cDNA library with sera from Chagas disease (B), were stratified based on their frequency. "Others" stands for cDNAs encoding ribosomal proteins identified as ESTs with a frequency lower than $2 \%$. The values correspond to the percentage of each clone regarding the total number of ribosomal proteins. Single asterisks highlight clones present in both groups. Double asterisks correspond to cDNAs identified only by serum reactivity. Ribosomal proteins account for 9 and $54 \%$ of the total number of clones obtained by EST and immunoscreening protocols, respectively. 
(Hoft et al. 1989). We are currently investigating whether the repetitive portion of $\mathrm{L} 23 \mathrm{a}$ also makes this protein a member of the group of ribosomal antigens recognized by antibodies present in human chagasic sera.

Given that the completion of the sequencing of the $T$. cruzi genome, conducted by The International $T$. cruzi Sequencing Consortium, is within sight, all the information about the estimated 10,000 genes harbored by this parasite will soon be available. Together with other EST studies, this amastigote EST survey represents an additional contribution to future studies on the characterization of genes that may have a potential application towards the development of new methods to control Chagas disease.

\section{ACKNOWLEDGMENTS}

To Rosiane A Silva and Dr Daniella C Bartholomeu for discussions and comments on the manuscript.

\section{REFERENCES}

Acosta-Serrano A, Almeida IC, Freitas-Junior L, Yoshida N, Schenkman S 2001. The mucin-like super-family of Trypanosoma cruzi: structure and functional roles. Mol Biochem Parasitol 114: 143-150.

Agüero F, Abdellah KB, Tekiel V, Sánchez DO, González A 2004. Generation and analysis of expressed sequence tags from Trypanosoma cruzi trypomastigotes and amastigotes cDNA libraries. Mol Biochem Parasitol 136: 221-225.

Altschul SF, Gish W, Miller W, Myers EW, Lipman DJ 1990. Basic local alignment search tool. J Mol Biol 215: 403-410.

Boscardin SB, Kinoshita SS, Fujimura AE, Rodrigues MM 2003. Immunization with cDNA expressed by amastigotes of Trypanosoma cruzi elicits protective immune response against experimental infection. Infect Immun 71: 2744-2757.

Brandão A, Urmenyi T, Rondinelli E, Gonzalez A, De Miranda AB, Degrave W 1997. Identification of transcribed sequences (ESTs) in the Trypanosoma cruzi genome project. Mem Inst Oswaldo Cruz 92: 863-866.

Brener Z 1973. Biology of Trypanosoma cruzi. Ann Rev Microbiol 27: 347-382

Chatterjee S, Singh S, Sohoni R, Kattige V, Deshpande C, Chiplunkar S, Kumar N, Sharma S 2000. Characterization of domains of the phosphoriboprotein P0 of Plasmodium falciparum. Mol Biochem Parasitol 107: 143-154.

Coetzee T, Herschlag D, Belfort M 1994. Escherichia coli proteins, including ribosomal protein $\mathrm{S} 12$, facilitates in vitro splicing of phage T4 introns by acting as RNA chaperones. Gene Dev 8: 11575-11565.

DaRocha WD, Bartholomeu DC, Macedo CD, Horta MF, CunhaNeto E, Donelson JE, Teixeira SM 2002. Characterization of cDNA clones encoding ribonucleoprotein antigens expressed in Trypanosoma cruzi amastigotes. Parasitol Res 88: 292-300.

Ewing B, Hillier L, Wendl MCM, Green P 1998. Base-calling of automated sequencer races using phred. I. Accuracy assessment. Genome Res 8: 175-185.

Frasch ACC 2001. Functional diversity in the Trans-sialidase and Mucin families in Trypanosoma cruzi. Parasitol Today 16: 282-285.

Hoft DF, Kim KS, Otsu K, Moser DR, Yost WJ, Blumin JH, Donelson JE, Kirchhoff LV 1989. Trypanosoma cruzi ex- presses diverse repetitive protein antigens. Infect Immun 57: 1959-1964.

Kaplan D, Ferrari I, Bergami PL, Mahler E, Levitus G, Chiale P, Hoebeke J, Van Regenmortel MHV, Levin MJ 1997. Antibodies to ribosomal $\mathrm{P}$ proteins of Trypanosoma cruzi in Chagas disease possess functional autoreactivity with heart tissue and differ from anti-P autoantibodies in lupus. Proc Nat Acad Sci USA 94: 10301-10306.

Levin MJ, Mesri E, Benarous R, Levitus G, Schijman A, LevyYeyati P, Chiale PA, Ruiz AM, Kahn A, Rosenbaum MB 1989. Identification of major Trypanosoma cruzi antigenic determinants in chronic Chagas' heart disease. Am J Trop Med Hyg 41: 530-538.

Low HP, Santos MAM, Wizel B, Tarleton RL 1998. Amastigote surface proteins of Trypanosoma cruzi are targets for $\mathrm{CD}^{+}$ CTL. J Immunol 160: 1817-1823.

Matsumoto TK, Hoshino-Schimizu S, Nakamura PM, Andrade HF, Umezawa ES 1993. High resolution of Trypanosoma cruzi amastigote antigen in serodiagnosis of different clinical forms of Chagas disease. J Clin Microbiol 31: 14861492.

Minning TA, Bua J, Garcia GA, McGraw RA, Tarleton RL 2003. Microarray profiling of gene expression during trypomastigote to amastigote transition in Trypanosoma cruzi. Mol Biochem Parasitol 131: 55-64.

Pais FS, DaRocha WD, Campos P, Cunha-Neto E, Teixeira SMR 2002. Human chagasic responses against $T$. cruzi recombinant antigens derived from ribonucleproteic complexes. Proceedings of the XXIX Annual Meeting on Basic Research in Chagas Disease Caxambu, MG.

Porcel BM, Tran AN, Tammi M, Nyarady Z, Rydaker M, Urmenyi TP, Rondinelli E, Pettersson U, Andersson B, Aslund L 2000. Gene survey of the pathogenic protozoan Trypanosoma cruzi. Genome Res 10: 1103-1107.

Recinos RF, Kirchhoff LV, Donelson JE 2001. Cell cycle expression of histone genes in Trypanosoma cruzi. Mol Biochem Parasitol 113: 215-222.

Requena JM, Alonso CA, Soto M 2000. Evolutionary conserved proteins as prominent immunogens during Leishmania infections. Parasitol Today 16: 246-250.

Skeiky YA, Benson DR, Elwasila M, Badaro R, Burns JM JR, Reed SG 1994. Antigens shared by Leishmania species and Trypanosoma cruzi: immunological comparison of the acidic ribosomal P0 proteins. Infect Immun 62: 1643-1651.

Teixeira SMR, Russell DG, Kirchhoff LV, Donelson JE 1994. A differentially expressed gene family encoding "amastin", a surface glycoprotein of Trypanosoma cruzi amastigotes. J Biol Chem 269: 20509-20516.

Vasconcelos AT, The Brazilian National Genome Project Consortium 2003. The complete genome sequence of Chromobacterium violaceum reveals remarkable and exploitable bacterial adaptability. Proc Nat Acad Sci USA 100: 1166011665.

Vazquez MP, Schijman AG, Levin MJ 1994. A short interspersed repetitive element provides a new 3' acceptor site for transsplicing in certain ribosomal P2 beta protein genes of Trypanosoma cruzi. Mol Biochem Parasitol 64: 327-336.

Verdun RE, Di Paolo N, Urmenyi TP, Rondinelli E, Frasch AC, Sanchez DO 1998. Gene discovery through expressed sequence Tag sequencing in Trypanosoma cruzi. Infect Immun 66: 5393-5398. 
